\title{
SIMPLE EXAMPLES OF THE DERIVATION OF AMPLITUDE EQUATIONS FOR SYSTEMS OF EQUATIONS POSSESSING BIFIURCATIONS
}

\author{
A. J. ROBERTS
}

(Received 25 October 1984))

\begin{abstract}
The method of Coullet and Spiegel [3], which derives ordinary differential equations describing the time evolution of a system of partial differential equations when the system is near critical, is applied to some simple problems. These problems serve to illustrate simply many features of the method.
\end{abstract}

\section{Introduction}

The evolution of a physical instability can often be described fairly simply in terms of the evolution of the amplitudes of certain dominant modes. Such a description is typically accurate only when the dominant modes are either slightly unstable or slightly damped. Coullet and Spiegel [3], henceforth denoted by CS, have developed a technique for systematically refining the mathematical description of the evolution to make it reflect more accurately the physical system.

The introduction of the paper by CS gives a description placing their method in relation to other methods which derive similar evolution equations. CS discuss and apply their method in its full generality. The aim of this paper is to provide an intermediary step in the understanding of the method, by applying it to some simple sets of differential equations; in doing so some minor flaws in the method are discovered.

Consequently, parts of this paper are virtually copied from CS, not to plagiarize what they wrote but to illustrate what they meant. Also, much of the numbering of equations in this paper is designed so that an equation in this paper can often

\footnotetext{
${ }^{1}$ Department of Applied Mathematics, University of Adelaide, Adelaide, S.A. 5000

(c) Copyright Australian Mathematical Society 1985, Serial-fee code 0334-2700/85
} 
be directly compared with the equation of the same number of CS. This has led to jumps in the numbering, although it is still monotonic.

Some terminology and background are introduced in Section 2, where a problem which can be solved exactly in closed form is analysed. Such exact solutions permit an examination of the relation between the typically asymptotic results of the method and the actual solutions. In Section 3 the method is applied to a problem which is not analytically solvable, and this forces the use of the full method. However, the problem is simple enough so that many of the complicated features of the full method appear here in a very simple form. Some concluding remarks are given in Section 4, while some side issues are discussed in the Appendices.

\section{A preliminary example}

Before proceeding to analyse an example which possesses most of the features generally present, we will examine a slightly simpler example. The methods of analysis used in this section are different to those of the general method. The main purpose of this section is to show the existence of the centre manifold and corresponding evolution equation, which are assumed to exist in the analysis of Section 3.

Consider the nonlinear autonomous pair of differential equations

$$
\begin{gathered}
\dot{\alpha}=\lambda \alpha-\alpha \beta, \\
\dot{\beta}=-\beta+\alpha^{2}-2 \beta^{2},
\end{gathered}
$$

where $\left({ }^{\circ}\right)$ denotes $d / d t$ and $\lambda$ is a parameter. This pair of equations was chosen because the trajectories of the solutions can be found analytically and hence some features of the asymptotic results we derive can be compared to the exact results. (See Appendix B for ways to derive other solvable equations). These equations are of the very general form examined by CS, namely

$$
\dot{\mathbf{U}}=M_{\lambda} \mathbf{U}+\mathbf{N}_{\lambda}(\mathbf{U})
$$

in which we identify

$$
\begin{aligned}
\mathbf{U} & =\left(\begin{array}{l}
\alpha \\
\beta
\end{array}\right), \\
M_{\lambda} & =\left(\begin{array}{rr}
\lambda & 0 \\
0 & -1
\end{array}\right), \\
\mathbf{N}_{\lambda}(\mathrm{U}) & =\left(\begin{array}{c}
-\alpha \beta \\
+\alpha^{2}-2 \beta^{2}
\end{array}\right) .
\end{aligned}
$$


All the linear terms have been grouped into the term $M_{\lambda} \mathrm{U}$, while $\mathrm{N}_{\lambda}(\mathrm{U})$ contains the nonlinear terms.

Equation (2.1) is homogeneous and hence $\mathbf{U}=\mathbf{0}$ is a possible solution. The questions then asked are: For what values of the parameter $\lambda$ is $\mathbf{U}=\mathbf{0}$ stable, and when $\mathbf{U}=\mathbf{0}$ is not stable, how does the system evolve?

When the initial conditions imply that the magnitude of $\mathbf{U}$ is small, the initial hehaviour is described by omitting the nontineẫ termins $N_{\lambda}(U)$. In the resuiting linear problem, we seek solutions of the form

$$
\mathbf{U}=e^{\eta t} \boldsymbol{\Phi} .
$$

This leads to the eigenvalue problem

$$
M_{\lambda} \Phi=\eta \Phi
$$

which, for the problem (2.1), has solutions, called normal modes, and corresponding characteristic value equations

$$
\begin{array}{ll}
\Phi_{1}=\left(\begin{array}{l}
1 \\
0
\end{array}\right), & P_{1}(\eta ; \lambda) \equiv \eta-\lambda=0, \\
\Phi_{2}=\left(\begin{array}{l}
0 \\
1
\end{array}\right), & P_{2}(\eta ; \lambda) \equiv \eta+1=0 .
\end{array}
$$

The complete characteristic value equation for the linear theory of $(2.1)$ is thus

$$
P(\eta ; \lambda)=(\eta-\lambda)(\eta+1)=0 .
$$

Observe that, for the value $\lambda=\lambda_{0}=0$, there are

$$
d=1
$$

roots of (2.4) with $\operatorname{Re}\{\eta\}=0$. Such a root and the associated mode $\Phi_{1}$ are described as critical. For $\lambda$ near 0 there will still be $d=1$ root with small $|\operatorname{Re}\{\eta\}|$ while the other root of (2.4) has $\operatorname{Re}\{\eta\} \leqslant \eta_{0}=-1$. A simplified asymptotic description of the time dependence of (2.1) occurs in the limit $\left|\lambda / \eta_{0}\right| \rightarrow 0$. This becomes possible because, for $\lambda$ near the critical set $\Sigma=\{0\}$, the evolution of the stable mode $\Phi_{2}$ (towards zero) occurs so quickly in comparison to the evolution of the critical mode $\Phi_{1}$ that the solution is predominantly composed of the critical mode.

\subsection{The critical case, $\lambda=0$}

We now proceed to derive such a simplified description for the special case when $\lambda$ is precisely the critical value 0 . $U$ can be written as a sum of critical modes and stable modes

$$
\mathbf{U}(t)=\alpha(t)\left(\begin{array}{l}
1 \\
0
\end{array}\right)+\beta(t)\left(\begin{array}{l}
0 \\
1
\end{array}\right)
$$


which, upon substitution into (2.1), yields precisely (2.1) with $\lambda=0$, namely

$$
\begin{aligned}
& \dot{\alpha}=-\alpha \beta, \\
& \dot{\beta}=-\beta+\alpha^{2}-2 \beta^{2} .
\end{aligned}
$$

The equations as originally stated are fortuitously in the requisite form. For comparison with CS we identify $J=0, L=-1, F=-\alpha \beta$ and $G=\alpha^{2}-2 \beta^{2}$.

Suppose we introduce the nonlinear transform

$$
\beta=b+B(\alpha),
$$

into (2.11), then on using (2.10) we find

$$
\dot{b}=\left(-b+\alpha \frac{d B}{d \alpha} b-4 B b-2 b^{2}\right)+\left(\alpha B \frac{d B}{d \alpha}-B+\alpha^{2}-2 B^{2}\right) .
$$

Clearly if we choose $B(\alpha)$ such that

$$
\alpha B \frac{d B}{d \alpha}-\beta+\alpha^{2}-2 \beta^{2}=0,
$$

then $b=0$ is a solution of (2.13), and hence $\beta=B(\alpha)$ defines an invariant manifold of (2.10-2.11). Writing (2.14) in the form

$$
\frac{d B}{d \alpha}=\frac{-B+\alpha^{2}-2 B^{2}}{-\alpha \beta},
$$

we observe that $\beta=B(\alpha)$ just describes a trajectory of the original system $(2.10-2.11)$. Once we have determined which trajectory is of interest, the evolution of the system along that trajectory will be described by substituting $\beta=B(\alpha)$ into (2.10). Integrating (2.14) is not simple, but upon rewriting as

$$
\alpha B d B+\left(-B+\alpha^{2}-2 B^{2}\right) d \alpha=0,
$$

we observe that this is proportional to the exact differential of

$$
\psi=\left(\frac{B}{\alpha^{2}}-1\right) \exp \left[\frac{1+2 B}{2 \alpha^{2}}\right] .
$$

Thus we find an implicit equation for $B$ as a function of $\alpha ; \psi$ acts as a constant of integration which determines the particular trajectory. For example, the trajectory $\psi=0$ is the manifold

$$
\beta=\alpha^{2},
$$

upon which the solution evolves according to

$$
\dot{\alpha}=F(\alpha, B(\alpha))=-\alpha^{3} .
$$

We now illustrate a minor difficulty overlooked by CS. CS proposed that the initial condition $\left.(d B / d \alpha)\right|_{\alpha=0}=0$ would uniquely determine the trajectory of interest. However, this example shows that this is not so. It is easy to derive

$$
B-\alpha^{2}\left\{1+\frac{\psi}{e} \exp \left[\frac{-1}{2 \alpha^{2}}\right]\right\}, \quad \alpha \rightarrow 0^{+},
$$


from which we readily deduce that $\left.(d B / d \alpha)\right|_{\alpha=0}=0$ for all trajectories, and this condition is thus insufficient to determine a unique trajectory. The difficulty appears to be unresolvable in this problem and perhaps can only be resolved for $\lambda>0$ when there is a unique trajectory passing through both the fixed point $\mathbf{U}=\mathbf{0}$ and the nearby stable fixed point.

However, for a general problem of this type, equation (2.14) has to be solved via an asymptotic analysis in powers of a. Analytic solutioñs like (2.15) âre generally not available and numerical solutions are extremely difficult to obtain, due to the presence of exponentially growing terms. In such an asymptotic series, the difference between different trajectories, being exponentially small, is insignificant. If an asymptotic analysis were to be done for this example problem (2.10-2.11), then the simplified description of the evolution of the solultion would thus be precisely (2.16).

\subsection{The case of general $\lambda$}

For values of $\lambda$ near the critical value 0 , we now suppose that an amplitude equation similar to (2.16b) governs the evolution of the system on a manifold similar to that given by $(2.16 \mathrm{a})$. That is, we propose

$$
\begin{aligned}
& \beta=B_{\lambda}(\alpha), \\
& \dot{\alpha}=J_{\lambda} \alpha+g_{\lambda}(\alpha),
\end{aligned}
$$

where $g_{\lambda}(\alpha)$ exclusively contains all the nonlinear terms in $\alpha$ and $J_{0}=0$. The task is then to find the appropriate $B_{\lambda}, J_{\lambda}$ and $g_{\lambda}$.

As a first approximation CS proposed that for $\lambda$ very close to the critical value, the only qualitatively important difference between (2.19) and (2.16) lies in the introduction of the linear term $J_{\lambda} \alpha$. Thus CS proposed that the evolution of (2.1) is approximated by

$$
\begin{gathered}
\beta=\alpha^{2}, \\
\dot{\alpha}=\mu(\lambda) \alpha-\alpha^{3},
\end{gathered}
$$

where we may readily verify that $\mu(\lambda)=\lambda$.

However, in this example we can do significantly better. In fact, all that needs to be done is to apply the method of Section 2.1 to the full problem. Upon substituting the nonlinear transformation (2.12) into equations (2.1), we find

$$
\dot{b}=\left(-b+\alpha \frac{d B}{d \alpha} b-4 B b-2 b^{2}\right)+\left(-\lambda a \frac{d B}{d \alpha}+\alpha B \frac{d B}{d \alpha}-B+\alpha^{2}-2 B^{2}\right) .
$$

Clearly, if we choose $B(\alpha)$ such that

$$
-\lambda \alpha \frac{d B}{d \alpha}+\alpha \beta \frac{d B}{d \alpha}-B+\alpha^{2}-2 B^{2}=0,
$$


then $b=0$ is a solution of (2.20) and hence $\beta=B(\alpha)$ is an invariant manifold of (2.1). Just as before, we may write (2.21) as

$$
\frac{d B}{d \alpha}=\frac{-B+\alpha^{2}-2 B^{2}}{\lambda \alpha-\alpha B},
$$

and observe that $\beta=B(\alpha)$ must describe a trajectory of the system (2.1).

As shall be seen in Section 3, equations corresponding to (2.22) will in general have to be solved via a double asymptotic series in both $\alpha$ and $\lambda$, when $\lambda \neq 0$. In this example we know the exact solution (2.16a), and so expect that (2.22) could be solved via an asymptotic expansion in $\lambda$, with coefficients which are functions of $\alpha$ (the leading term being $\beta=\alpha^{2}$; see Appendix $A$ for such an example). However, this is not necessary here, as (2.22) is in fact integrable. The differential form

$$
(-\lambda \alpha+\alpha B) d B+\left(-B+\alpha^{2}-2 B^{2}\right) d \alpha=0,
$$

is proportional to the exact differential of

$$
\psi=\left(\frac{B}{\alpha^{2}}-\frac{1}{1+2 \lambda}\right)\left|1-\lambda\left(\frac{1+2 B}{\alpha^{2}}\right)\right|^{-1-1 / 2 \lambda}
$$

which reduces to (2.15) in the limit as $\lambda \rightarrow 0$.

For $\lambda>0$ there is a unique trajectory through the origin, namely $\psi=0$, which also passes through the finite amplitude fixed point (as the fixed point is stable). Thus the manifold upon which the solution evolves is

$$
\beta=\frac{\alpha^{2}}{1+2 \lambda},
$$

where the evolution of $\alpha$ is governed by substituting (2.24a) into (2.1a), namely

$$
\alpha=\lambda \alpha-\frac{1}{1+2 \lambda} \alpha^{3}
$$

Furthermore, this manifold is stable, as the evolution along neighbouring trajectories is such as to bring $(\alpha, \beta)$ closer to this line. For $\lambda<0$, all trajectories pass through the stable fixed point at the origin, and hence the problem of choosing the appropriate trajectory is as difficult as for the case $\lambda=0$. In fact, the problem is worse, because

$$
B \sim \frac{\alpha^{2}}{1+2 \lambda}+\psi|\lambda|^{1+1 / 2 \lambda}|\alpha|^{-1 / \lambda} \text { as } \alpha \rightarrow 0,
$$

which shows that the difference between different trajectories is now not exponentially small, and would become significant in an asymptotic treatment of problems like (2.1) at order $\alpha^{-1 / \lambda}$. 


\section{An example application of the general method}

Applying the general method of CS to the problem discussed in Section 2 would lead to the near-trivial results expressed in (2.16) and (2.24). Thus in this section the similarly simple problem

$$
\begin{aligned}
& \dot{\alpha}=\lambda \alpha-\alpha \beta, \\
& \dot{\beta}=-\beta+\alpha^{2},
\end{aligned}
$$

is discussed. Although the solutions of (3.1) near the origin behave similarly to those of (2.1), no analytic solutions to (3.1) are known and a simplified description of the evolution of the solutions relies on a fully-fledged asymptotic expansion.

Evolution equations for (3.1) have been derived via the alternative method of reconstitution (Roberts [4]); thus these two methods for deriving more accurate evolution equations can be compared.

Following CS we propose that a stable solution of (3.1) can be expressed as

$$
\mathbf{U}(t ; \lambda)=\mathbf{V}(A ; \lambda)
$$

where $A$ satisfies the ordinary differential (evolution) equation

$$
\dot{A}=G(A ; \lambda)
$$

(and $\mathbf{U}$ is the vector of unknowns $\left.(\alpha, \beta)^{T}\right)$. Just as in Section 2, the simplest problem to analyse is the fundamental $\lambda=0$ problem (see Section 3.1). The behaviour for $\lambda$ very near zero can then be deduced simply by modifying those terms of $G(A ; 0)$ which are linear in $A$. However, for larger values of $\lambda$ (but still small) a more extensive analysis has to be done (see Section 3.2).

\subsection{The critical case, $\lambda=0$}

The linear theory for (3.1) is precisely the same as that for (2.1) and shows that the solution space can be spanned by a vector from the critical space, namely $\Phi_{1}=(1,0)^{T}$, and a vector from the stable space, namely $\Phi_{2}=(0,1)^{T}$. Thus we may write

$$
\begin{aligned}
\mathbf{V}(A ; 0) & =F(A)\left(\begin{array}{l}
1 \\
0
\end{array}\right)+\mathbf{W}(A), \\
& =F(A) \Phi_{1}+\left(\begin{array}{c}
0 \\
W(A)
\end{array}\right),
\end{aligned}
$$

so that $\mathbf{W}(A)$ lies entirely in the stable space.

We decompose $G(A ; 0)$ into its linear and nonlinear parts; thus $(3.2 \mathrm{~b})$ becomes

$$
\dot{A}=J_{0} A+g(A) \text {, }
$$

where $g(A)$ is strictly nonlinear and the linear analysis tells us that $J_{0}=0$. Similarly, $F(A)$ can be decomposed into

$$
F=A+\chi(A)
$$


where $\chi(A)$ is strictly nonlinear and where the linear term $A$ has been chosen to match the linear theory of (2.1). The same line of argument indicates that $W$ must be a strictly nonlinear function of $A$.

To reduce unnecessary complication we now depart slightly from $C S$ and henceforth set $\chi=0$. Allowing nonzero $\chi$ just allows us to define the amplitude $A$ to be any function of $\alpha$ and $\beta$ we like (subject to the constraint that $A-\alpha$ as $\alpha, \beta \rightarrow 0$ with $\beta \propto \alpha^{2}$ ). This freedom is not needed here and so we choose $A=\alpha$, i.e. $\chi=0$.

Substituting (3.2a), (3.3), (3.4) and (3.5) into (3.1) and grouping all the terms involving $\mathbf{W}$ on the left-hand side, we arrive at

$$
L \mathbf{W}-\hat{\mathbf{N}}(\mathbf{W})=M \Phi_{1} A-J_{0} A \Phi_{1}-g \Phi_{1}+\mathbf{N}\left(A \Phi_{1}\right),
$$

where in this problem

and

$$
\begin{aligned}
L & =J_{0} A \frac{\partial}{\partial A}-M, \\
M & =\left(\begin{array}{cc}
0 & 0 \\
0 & -1
\end{array}\right), \\
\mathbf{N}\left[\left(\begin{array}{l}
\alpha \\
\beta
\end{array}\right)\right] & =\left(\begin{array}{c}
-\alpha \beta \\
\alpha^{2}
\end{array}\right),
\end{aligned}
$$

(here $L$ is a matrix/operator, whereas in Section 2 it was a number). Equation (3.6) has been put into a form which is very close to the corresponding equation in CS, the differences being due to the omission of terms involving $\chi$. This close correspondence is important, as the general method is based upon this form of equation. However, for this problem, (3.6) is actually more simple than it first appears as $J_{0}=0$ and $\Phi_{1}=(1,0)^{T}$.

Since $\mathrm{W}$ is a strictly nonlinear function of $A$, the terms linear in $A$ on the right-hand side must cancel, hence

$$
M \Phi_{1}=J_{0} \Phi_{1}
$$

That this equation is satisfied just verifies that the choices of $M, J_{0}$ and $\Phi_{1}$ are correct. Equation (3.8) looks very much like, and can be thought of as, an eigenproblem for $J_{0}$ and $\Phi_{1}$. It can be written down directly from the linear terms in equations (3.1) (with $\lambda=0$ ). In general, to derive eigenvectors in the critical space, we must additionally require that all the eigenvalues of $J_{0}$ (itself a matrix in general) be zero. Where the critical space is one-dimensional, as it is here, this just requires $J_{0}=0$. When the critical space is of higher dimension (e.g. CS discuss the two-dimensional case) then there is some redundancy, which is resolved by choosing $J_{0}$ in Jordan form. Equation (3.6) now becomes

$$
L \mathbf{W}=\mathbf{N}\left(A \Phi_{1}+\mathbf{W}\right)-g \frac{\partial \mathbf{W}}{\partial A}-g \Phi_{1},
$$


(note that $\Xi$, as used by $C S$, is the same as $\mathbf{W}$, since we have taken $\chi=0$ ). The strategy is to choose $g$ so that (3.10) is solvable and, given $g$, to find the corresponding $\mathbf{W}$.

The two functions $g(A)$ and $\mathbf{W}(A)$ are determined together in terms of their Taylor series. Introducing

$$
\begin{gathered}
\overline{\mathbf{W}}(A)=\sum_{k=z}^{\infty} \overline{\mathrm{W}}_{k} A^{k}, \\
g(A)=\sum_{k=z}^{\infty} g_{k} A^{k},
\end{gathered}
$$

into (3.10), and equating like powers of $A$ we obtain the sequence of linear inhomogeneous equations

$$
L \mathbf{W}_{k}=\mathbf{I}_{k}-g_{k} \Phi_{1},
$$

where the nonlinear terms have been grouped into $\mathbf{I}_{k}$, which depends only on quantities with lower $k$. The treatment now becomes much simpler than that described in CS, because the critical space is only one-dimensional, and hence $L$ is simply a matrix rather than a matrix/operator as in the general higher-dimensional case.

In order to be able to solve (3.13) for $\mathbf{W}_{k}$, the right-hand side must be orthogonal to the solution, $\mathbf{Z}$, of the homogeneous adjoint linear problem, namely

$$
L^{+} \mathbf{Z}=\mathbf{0} \text {. }
$$

Here

$$
L^{+}=L=\left(\begin{array}{ll}
0 & 0 \\
0 & 1
\end{array}\right)
$$

and hence

$$
\mathbf{Z}=\left(\begin{array}{l}
1 \\
0
\end{array}\right)
$$

Thus, provided

$$
\mathbf{Z}^{T}\left(\mathbf{I}_{k}-g_{k} \Phi_{1}\right)=0
$$

equation (3.13) is solvable. This condition, and its analogues in other problems, are termed solvability conditions, and are used to determine $g_{k}$. Equation (3.13) is then used to calculate $W_{k}$, with the additional constraint that $\mathbf{W}$ lie entirely in the stable space; hence $\mathrm{W}_{k}=\left(0, W_{k}\right)^{T}$. 
Here, the first few members of the sequence of equations (3.13) are just

$$
\begin{aligned}
& L \mathbf{W}_{2}=\left(\begin{array}{l}
0 \\
1
\end{array}\right)-\left(\begin{array}{l}
1 \\
0
\end{array}\right) g_{2}, \\
& L \mathbf{W}_{3}=-\left(\begin{array}{c}
W_{2} \\
2 W_{2} g_{2}
\end{array}\right)-\left(\begin{array}{l}
1 \\
0
\end{array}\right) g_{3}, \\
& L \mathbf{W}_{4}=-\left(\begin{array}{c}
W_{3} \\
2 W_{2} g_{3}+3 W_{3} g_{2}
\end{array}\right)-\left(\begin{array}{l}
1 \\
0
\end{array}\right) g_{4}, \\
& L \mathbf{W}_{5}=-\left(\begin{array}{c}
2 W_{4} \\
2 g_{4}+3 W_{3} g_{3}+4 W_{4} g_{2}
\end{array}\right)-\left(\begin{array}{l}
1 \\
0
\end{array}\right) g_{5} .
\end{aligned}
$$

Applying the solvability condition (3.26) to (3.27a), we find that

$$
g_{2}=0 \text {, }
$$

and solving (3.27a) gives

$$
\mathbf{W}_{2}=\left(\begin{array}{c}
0 \\
W_{2}
\end{array}\right)=\left(\begin{array}{l}
0 \\
1
\end{array}\right)
$$

Continuing on to solve the other equations in sequence in the same manner, we find

$$
\begin{array}{ll}
g_{3}=-1, & W_{3}=0, \\
g_{4}=0, & W_{4}=2, \\
g_{5}=-2, & W_{5}=0 .
\end{array}
$$

Thus the amplitude equation (3.4) is (since $J_{0}=0$ )

$$
\dot{A}=g(A)-A^{3}-2 A^{5}, \quad A \rightarrow 0 ;
$$

while the centre manifold upon which this evolution takes place is

$$
\mathbf{U}=\mathbf{V}(A)=\left(\begin{array}{c}
A \\
W(A)
\end{array}\right)-\left(\begin{array}{c}
A \\
A^{2}+2 A^{4}
\end{array}\right), \quad A \rightarrow 0 .
$$

In fact, it is easy to extend this analysis to much higher order and find that

$$
g(A)=-A W(A),
$$

where

$$
\begin{aligned}
W(A)- & A^{2}+2 A^{4}+12 A^{6}+112 A^{8}+1360 A^{10}+19872 A^{12} \\
& +335104 A^{14}+6359040 A^{16}+133560576 A^{18} \\
& +3069007360 A^{20}+76493880320 A^{22}, \quad A \rightarrow 0,
\end{aligned}
$$

which is divergent, and just goes to show the truely asymptotic nature of the analysis. 


\subsection{The case of general $\lambda$}

Just as in Section 2.2, we may argue that for very small values of $\lambda$ the only qualitatively important change to the solution (3.32) and (3.33) is the introduction of a linear term in the amplitude equation that controls the stability of the fixed point $\mathbf{U}=\mathbf{0}$. Thus we would conclude that

$$
\dot{A} \approx \lambda A-A^{3}-2 A^{5},
$$

and

$$
\mathrm{U} \approx\left(\begin{array}{c}
A \\
A^{2}+2 A^{4}
\end{array}\right)
$$

describe the important solutions of (3.1). However, a more systematic approach can be used (Appendix C in CS) which finds additional corrections to $g(A)$ and $W(A)$, and it is this approach which is applied to (3.1) in this section.

Equation (3.1) can be written in the general form (2.1c) where here

$$
\begin{aligned}
\mathbf{U} & =\left(\begin{array}{l}
\boldsymbol{\alpha} \\
\boldsymbol{\beta}
\end{array}\right), \\
M_{\lambda} & =\left(\begin{array}{cc}
\lambda & 0 \\
0 & -1
\end{array}\right), \\
\mathbf{N}_{\lambda}(\mathbf{U}) & =\left(\begin{array}{c}
\alpha \boldsymbol{\beta} \\
\alpha^{2}
\end{array}\right) .
\end{aligned}
$$

We now substitute the usual ansatz for the solutions

$$
\begin{gathered}
\mathrm{U}(t, \lambda)=\mathrm{V}(A, \lambda), \\
\dot{A}=G(A, \lambda),
\end{gathered}
$$

and rearrange the resultant equation,

$$
G \frac{\partial \mathbf{V}}{\partial A}=M_{\lambda} \mathbf{V}+\mathbf{N}_{\lambda}(\mathbf{V})
$$

into a form similar to that of (3.10), namely

$$
L \mathbf{V}=\left(M_{\lambda}-M_{0}\right) \mathbf{V}+N_{\lambda}(\mathbf{V})-\left(G-G_{1}^{0} A\right) \frac{\partial \mathbf{V}}{\partial A}
$$

where, as before

$$
\begin{aligned}
G_{1}^{0} & =J_{0}=0, \\
L & =\left(G_{1}^{0} A \frac{\partial}{\partial A}-M_{0}\right)=\left(\begin{array}{ll}
0 & 0 \\
0 & 1
\end{array}\right) .
\end{aligned}
$$

Observe that all the linear terms are grouped on the left-hand side of equation (3.41) while all the nonlinear terms are on the right-hand side; the dependence upon $\lambda-\lambda_{0}=\lambda$ is also taken into account when deciding upon the linearity of 
any term. This separation of linear and nonlinear terms is required for the method.

We now proceed by a slightly different, but equivalent, formalism to that used in Section 3.1. Assume that the parameter $\lambda$ is near the critical value $\lambda_{0}=0$, and hence we can develop the functions as Taylor series in $\lambda$, i.e.

$$
\begin{aligned}
& \mathbf{v}(A, \lambda)=\sum_{l=0}^{\infty} \lambda^{l} \mathbf{V}^{\prime}(A), \\
& G(A, \lambda)=\sum_{l=0}^{\infty} \lambda^{l} G^{l}(A) .
\end{aligned}
$$

Then, just as in Section 3.1, we develop in powers of $A$ :

$$
\begin{aligned}
& V^{\prime}(A)=\sum_{k=1}^{\infty} A^{k} \mathbf{V}_{k}^{l}, \\
& G^{\prime}(A)=\sum_{k=1}^{\infty} A^{k} G_{k}^{l} .
\end{aligned}
$$

Note that we have diverged slightly from the notation used by CS in that the dependence upon $A$ is explicit (with $V_{k}^{l}$ and $G_{k}^{l}$ constants) rather than implicit in $\mathbf{V}_{k}^{l}$ and $G_{k}^{l}$. This is reasonable here as there is only one amplitude function $A$. Substituting the two double Taylor series (3.42) and (3.43) into (3.41) and equating like powers of $\lambda^{\prime} A^{k}$, we obtain a set of equations of the form

$$
\begin{aligned}
L \mathbf{V}_{1}^{0} & =\mathbf{0}, \\
L \mathbf{V}_{k}^{\prime} & =\mathbf{I}_{k}^{\prime}-G_{k}^{\prime} \mathbf{V}_{1}^{0}, \quad(k, l) \neq(1,0),
\end{aligned}
$$

where $\mathbf{I}_{k}^{\prime}$ only depends upon quantities with a lower value of $k$ or $l$.

Just as in Section 3.1, equation (3.44a) is very like an eigenproblem, here having the solution

$$
\mathbf{V}_{1}=\Phi_{1}^{0}=\left(\begin{array}{l}
1 \\
0
\end{array}\right), \quad G_{1}^{0}=0 .
$$

For successively higher values for $k$ or $l$, equation (3.44b) can be solved for $V_{k}^{l}$, but only if the right-hand side satisfies the solvability condition

$$
\mathbf{Z}^{T}\left(\mathbf{I}_{k}^{\prime}-G_{k}^{l} \mathbf{V}_{1}^{0}\right)=0, \quad \mathbf{Z}=\left(\begin{array}{l}
1 \\
0
\end{array}\right),
$$

which serves to determine $G_{k}^{l}$. Additionally, we also require that $\mathbf{V}_{k}^{l}$ lie entirely in the stable space; here we take

$$
\mathbf{V}_{k}^{\prime}=\left(\begin{array}{c}
0 \\
V_{k}^{\prime}
\end{array}\right), \quad(k, l) \neq(1,0)
$$

[Note that CS's introduction of $\chi$, which for simplicity we have neglected, is exactly equivalent to relaxing this arbitrary constraint. To make $V_{k}^{l}$ unique, a constraint is needed, but this could be something more useful, e.g. one possibility 
is to define $A$ to be the distance in $(\alpha, \beta)$-plane from $(0,0)$, rather than defining $A$ to be $\alpha$ which is implicit in (3.47).] Using (3.47) we can determine that here

$$
\mathbf{I}_{k}^{\prime}=\left(\begin{array}{c}
\delta_{l 1} \boldsymbol{\delta}_{k 1}-\left(1-\delta_{10} \boldsymbol{\delta}_{k 2}\right) V_{k-1}^{l} \\
\delta_{10} \delta_{k 2}-\sum_{i=0}^{l} \sum_{j=1}^{k} j V_{j}^{\prime} G_{k+1-\jmath}^{l-i} \\
(i, j) \notin\{(0,1),(i, k)\}
\end{array}\right),
$$

where $\delta_{i j}$ is the Kronecker delta. The calculations now are straightforward but a little tedious (observe that the $l=0$ sequence of equations are precisely those solved in Section 3.1). The solutions to (3.44b) for $0 \leqslant l \leqslant 2$ and $1 \leqslant k \leqslant 5$ lead to the following evolution equation

$$
\begin{aligned}
\dot{A}=G(A, \lambda)=\lambda A & -\left(1-2 \lambda+4 \lambda^{2}\right) A^{3}-2\left(1-8 \lambda+44 \lambda^{2}\right) A^{5} \\
& +O\left(A^{5}, \lambda A^{5}, \lambda^{2} A^{5}, \lambda^{3}\right),
\end{aligned}
$$

governing the solution

$$
\mathbf{U}=\mathbf{V}(A, \lambda)=\left(\begin{array}{c}
A \\
W(A, \lambda)
\end{array}\right)
$$

where

$$
\begin{aligned}
W= & \left(1-2 \lambda+4 \lambda^{2}\right) A^{2}+2\left(1-8 \lambda+44 \lambda^{2}\right) A^{4} \\
& +O\left(A^{5}, \lambda A^{5}, \lambda^{2} A^{5}, \lambda^{3}\right) .
\end{aligned}
$$

\section{Concluding remarks}

In the preceding sections, the method proposed by Coullet and Spiegel [3] has been applied to perhaps the simplest nontrivial examples. These have illustrated much, but not all, of the technique.

In addition, difficulties in determining precisely the required centre manifold, overlooked by Coullet and Spiegel, were found in the exactly solvable example of Section 2. Also, the example treated in Section 3 (and the example in Appendix A) are sufficiently simple to show the truly asymptotic nature of the analysis. It is interesting to note here that convergence acceleration techniques when applied to divergent sums, such as (3.35) and (A12), can produce a reasonable and accurate value for the sum over a wide range of the parameter (see Bender and Orszag [2] Chapter 8). However, when applied to divergent sums like (3.35) and (A12) they will converge only for $A^{2}=\alpha^{2} \leqslant 0$ (see Bender and Orszag [2] Section 8.6). Thus, although convergence acceleration techniques may be useful in other problems of this type, they are of no use here. The results here are at best asymptotic. On 
comparing this application of the method of Coullet and Spiegel [3] and the application of the technique of reconstitution (Roberts [4]) to equation (3.1) we find the results, where they overlap, are precisely the same. For example, the terms common to the descriptions of the centre manifold, equations $(3.49 \mathrm{c})$ here and equation (4.6c) in Roberts [4], are the same. The descriptions differ only in which terms have been obtained.

Also, observe that results obtained via the method of Coullet and Spiegel are obtained with no redundant algebra as is found in the method of reconstitution. However, reconstitution gives much more flexibility in the form of the resulting description of the evolution of the solutions; this may be valuable. Perhaps the method of Coullet and Spiegel can be generalized to give more general forms of results.

\section{Appendix A}

\section{A problem involving an asymptotic expansion in only $\mathbf{A}$}

In this appendix we illustrate the type of singular functions involved in solving differential equations like (2.1) or (3.1). In particular, the sort of nonanalytic behaviour encountered here at the origin is expected to be fairly typical in the analysis of more complex bifurcation problems.

Consider the pair of equations

$$
\begin{aligned}
& \dot{\alpha}=\lambda \alpha-(1+\lambda) \alpha \beta, \\
& \dot{\beta}=-\beta+\alpha^{2}-2 \beta^{2},
\end{aligned}
$$

which reduce to (2.10) and (2.11) for $\lambda=0$, but which for nonzero $\lambda$ are a slightly modified version of the analytically solvable (2.1). Since the trajectories of (A1) and (A2) are known exactly for $\lambda=0$, given by (2.15), then we shall approximately solve (A1) and (A2) for nonzero $\lambda$ by constructing the first two terms in an asymptotic expansion in powers of $\lambda$.

The nonzero- $\lambda$ analysis of (2.1) given in Section 2.2 indicates that the trajectory of interest for $\lambda=0$ is

$$
\beta=B_{0}(\alpha)=\alpha^{2} .
$$

Since (2.1) and (A1) and (A2) reduce to the same equations for $\lambda=0$, we then presume that (A3) gives the first approximation to the centre manifold of equations (A1) and (A2). (The alternatives differ by an exponentially small 
amount and would not significantly affect the following analysis.) We now seek $B_{1}(\alpha)$ such that

$$
\begin{aligned}
\beta & =B(\alpha)=B_{0}(\alpha)+\lambda B_{1}(\alpha)+O\left(\lambda^{2}\right) \\
& =\alpha^{2}+\lambda B_{1}(\alpha)+O\left(\lambda^{2}\right),
\end{aligned}
$$

is a better description of the centre manifold. Substituting (A4) into the equation prescribing the trajectories of (A1) and (A2), namely

$$
[\lambda \alpha-(1+\lambda) \alpha \beta] \frac{d \beta}{d \alpha}=\left[-\beta+\alpha^{2}-2 \beta^{2}\right],
$$

and ignoring terms of $O\left(\lambda^{2}\right)$ we find that $B_{1}(\alpha)$ must satisfy

$$
\alpha^{3} \frac{d B_{1}}{d \alpha}-\left(2 \alpha^{2}+1\right) B_{1}=2 \alpha^{2}-2 \alpha^{4} .
$$

Solving this first order differential equation we find

$$
B_{1}=\alpha^{2} e^{-1 / 2 \alpha^{2}} \int\left(\frac{2}{\alpha^{3}}-\frac{2}{\alpha}\right) e^{1 / 2 \alpha^{2}} d \alpha,
$$

which upon substituting $x=1 / 2 \alpha^{2}$ becomes

$$
B_{1}=\alpha^{2} e^{-1 / 2 \alpha^{2}} \int\left(-2+\frac{1}{x}\right) e^{x} d x,
$$

and hence

$$
B_{1}=-2 \alpha^{2}+\alpha^{2} e^{-1 / 2 \alpha^{2}}\left[\operatorname{Ei}\left(\frac{1}{2 \alpha^{2}}\right)+C\right],
$$

where $C$ is an arbitrary constant of integration and

$$
\operatorname{Ei}(X)=\int_{-\infty}^{X} \frac{e^{x}}{x} d x
$$

is the exponential integral as defined in Abramowitz and Stegun [1]. Thus the centre manifold is

$$
\beta=\alpha^{2}-\lambda \alpha^{2}\left\{2-e^{-1 / 2 \alpha^{2}}\left[\operatorname{Ei}\left(1 / 2 \alpha^{2}\right)+C\right]\right\}+O\left(\lambda^{2}\right),
$$

Thus the singularity in the manifold at $\alpha=0$ is extremely complicated, behaving like $e^{-1 / 2 \alpha^{2}} \operatorname{Ei}\left(1 / 2 \alpha^{2}\right)$.

However, the asymptotic expansion about $\alpha=0$ (for positive $\alpha$ ) is a relatively simple expression. Using the result

$$
\operatorname{Ei}(X) \sim \frac{1}{X} e^{X} \sum_{n=0}^{\infty} \frac{n !}{X^{n}} \quad \text { as } X \rightarrow+\infty
$$

we find

$$
\beta-\alpha^{2}-\lambda \alpha^{2}\left\{2-2 \alpha^{2} \sum_{n=0}^{\infty} 2^{n} n ! \alpha^{2 n}\right\}+O\left(\lambda^{2}\right) \quad \text { as } \alpha \rightarrow 0,
$$


which is the result that would be obtained if an asymptotic treatment of (A1) and (A2), like that developed in Section 3 (see equation (3.35)), were to be done.

The arbitrary constant in (A10), $C$, multiplies an exponentially small term and is therefore ignorable. In fact, choosing a value for $C$ would seem to as difficult as the problem of determining an appropriate trajectory for the $\lambda=0$ centre manifold, discussed in Section 2.1

\section{Appendix B}

\section{The derivation of solvable pairs of differential equations}

The first step in the development of this paper was to find a system of equations that were analytically solvable, so that the asymptotic results could be compared to the exact results. The pair of equations (3.1) have long been recognised informally as the model simple set of equations to illustrate bifurcation. Thus, the initial search is for strictly nonlinear functions $F$ and $G$ such that

$$
\begin{aligned}
& \dot{\alpha}=F(\alpha, \beta), \\
& \dot{\beta}=-\beta+G(\alpha, \beta),
\end{aligned}
$$

are analytically solvable; that is, we wish to solve the first-order differential equation

$$
\frac{d \beta}{d \alpha}=\frac{-\beta+G(\alpha, \beta)}{F(\alpha, \beta)} .
$$

Investigations of the possible use of standard techniques to solve (B3) failed to produce satisfactory forms for the functions $F$ and $G$. The only possibility is if (B3) is exact, that is

$$
\frac{d \beta}{d \alpha}=\frac{-\beta+G}{F}=\frac{\partial \psi / \partial \alpha}{-\partial \psi / \partial \beta},
$$

for some $\psi$, a function of $\alpha$ and $\beta$. The asymptotic behaviour of the trajectories of the solutions of (3.1) for small $\alpha$ and $\beta$ then suggests that we try

$$
\psi=\beta \exp \left(\frac{1}{2 \alpha^{2}}\right)
$$

However, this just leads to a decoupled pair of ordinary differential equations. Much trial and error then leads through

$$
\psi=\left(\beta-\alpha^{2}\right) \exp \left(\frac{1}{2 \alpha^{2}}\right)
$$


which couples the $\beta$ equations to $\alpha$ but still leaves the $\alpha$ equation solely in terms of $\alpha$, to

$$
\psi=\left(\beta-\alpha^{2}\right) \exp \left(\frac{1+2 \beta}{2 \alpha^{2}}\right),
$$

which at last couples the equations together in a manner similar to (3.1). Then, to simplify the resulting pair of ordinary differential equations, upon dividing the right-hand side of (B7) by $\overline{\boldsymbol{u}}^{2}$, we arrive at a $\dot{\psi}$ as specified by $(2.15)$, which gives the solvable pair of ordinary differential equations (2.1) (with $\lambda=0$ ).

The problem then is to modify (2.15) so as to introduce a dependence upon the parameter $\lambda$. Again, the asymptotic behaviour of solutions to (3.1) gives an indication of how to proceed. For $\lambda=0$, the trajectories are given by

$$
\beta-\psi \exp \left[-1 / 2 \alpha^{2}\right], \quad \alpha \rightarrow 0,
$$

while for nonzero $\lambda$, the trajectories are given by

$$
\beta \sim \psi|\alpha|^{-1 / \lambda}, \quad \alpha \rightarrow 0,
$$

which suggests that the exponential in (2.15) should be replaced by something raised to a power proportional to $1 / \lambda$. Remembering that

$$
e^{x}=\lim _{\mu \rightarrow 0}(1+\mu x)^{1 / \mu},
$$

we examine

$$
\psi=\left(\frac{\beta}{\alpha^{2}}-1\right)\left[1-\frac{\lambda(1+2 \beta)}{\alpha^{2}}\right]^{-1 / 2 \lambda},
$$

and find that this does indeed produce a pair of equations of the same form as (3.1), but with more nonlinear terms. Modifying the various coefficients in (B11) leads to equation (2.23), which describes the trajectories of the solutions to (2.1).

Other solvable differential equations with other requirements can be found by appropriately varying the forms of (B11) and (2.23). To do this successfully, an understanding of the form of (B11) or (2.23) is needed. Rewriting (2.23) as

$$
\psi=\left(\alpha^{2}\right)^{1 / 2 \lambda}\left(\beta-\frac{\alpha^{2}}{1+2 \lambda}\right)\left[\alpha^{2}-\lambda(1+2 \beta)\right]^{-1-1 / 2 \lambda},
$$

we can write down three special trajectories of the solutions to (2.1), namely

$$
\begin{aligned}
\alpha^{2} & =0, \\
\beta-\frac{\alpha^{2}}{1+2 \lambda} & =0, \\
\alpha^{2}-\lambda(1+2 \beta) & =0 .
\end{aligned}
$$

In the $\left(\alpha^{2}, \beta\right)$-plane the pairwise intersections of these trajectories give the fixed points of (2.1), namely

$$
\left(\alpha^{2}, \beta\right)=\left(\lambda+2 \lambda^{2}, \lambda\right), \quad\left(0,-\frac{1}{2}\right) \text { and }(0,0) .
$$


Thus the recipe to derive solvable differential equations would appear to be as follows. Choose the location of the fixed points, including the functional dependence upon $\lambda$. Then write down the equations of those lines whose pairwise intersections give the fixed points; these lines will become trajectories of the solutions. Then the product of each of the expressions forming each equation, raised to the appropriate power to give the desired behaviour near each fixed point, gives the equation for all the trajectories. Lastly, form the exact differential, and write down the pair of solvable ordinary differential equations.

\section{References}

[1] M. Abramowitz and I. A. Stegun, Handbook of mathematical functions (Dover, New York, 1965).

[2] C. M. Bender and S. A. Orszag, Advanced mathematical methods for scientssts and engineers (McGraw-Hill, New York, 1978).

[3] P. H. Coullet and E. A. Spiegel, "Amplitud equations for systems with competing instabilities", SIAM J. Appl. Math. 43 (1983), 776-821.

[4] A. J. Roberts, "An introduction to the technique of reconstitution", SIAM J. Math. Anal. (1985), (to appear). 